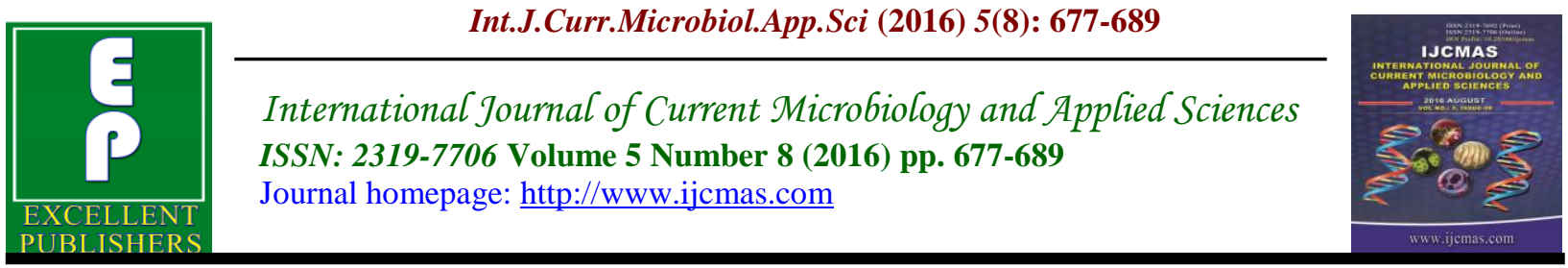

Original Research Article

http://dx.doi.org/10.20546/ijcmas.2016.508.077

\title{
Effect of Bisphenol-A on Brain Tissue in Pregnant Rat
}

\author{
T. Geetharathan* \\ Department of Biotechnology, Sri Padmavati Mahila Visvavidyalayam, \\ Tirupati-517502, Andhra Pradesh, India \\ *Corresponding author
}

Keywords

BPA,

Pregnant rat,

Brain,

Cerebellum,

MDA, GSH.

Article Info

Accepted:

28 July 2016

Available Online:

10 August 2016

\section{A B S T R A C T}

For more than ten years, there has been a scientific and journalistic controversy whether BPA causes adverse effects on human. Humans are routinely exposed to BPA, an estrogenic chemical present in food and beverage containers, dental composites, and many products in the home and workplace. In order to study the effects of BPA on the organ of pregnant rat brain, the albino rats were administrated to low\& high concentrations of BPA $(50 \mathrm{mg} / \mathrm{kg}$. d \& $500 \mathrm{mg} / \mathrm{kg}$. d). We aimed to study the toxic effect of BPA on the body \& brain weight, developmental structure of cerebellum in albino pregnant rat. After 8 days (Doses were administrated gestational day $8^{\text {th }}$ to $15^{\text {th }}$ ), the antioxidant enzymes, superoxide dismutase (SOD), catalase (CAT), malondialdehyde (MDA) and glutathione (GSH) concentrations in the organ was measured. Results showed that the MDA contents were increased with the added concentration of the BPA. But the SOD, CAT, GSH content was decreased. BPA induction also caused histopathological changes in the rat brain and cerebellum. All BPA treatment also revealed an increase in DNA fragmentation as evidenced by an increase in concentration of dose. It is indicated BPA can induce the rat's oxidative damage and the effect is much easier for the brain.

\section{Introduction}

Among many stabilizers of plastics, bisphenol-A (BPA) is a popular stabilizer that mimics the actions of estrogen and affects the reproduction, endocrine glands in vivo and in vitro (Kuiper et al., 1998). Animal studies have shown that in utero exposure to BPA produces prenatal and postnatal adverse effects on multiple tissues, including the brain (Richter et al., 2007). Prenatal BPA exposure affects brain development, sexual differentiation, social and anxiety-like behavior, and learning (or) memory (Kundakovic et al., 2011). In humans, emerging evidence for BPAassociated disruption to neurodevelopment is consistent with the rodent data and has revealed sex-specific effects of gestational BPA levels on emotional regulation and aggression in children (Perera et al., (2012). Growing evidence suggests that BPA is associated with oxidative stress (Sui et al., 2012). Indeed, it has been revealed that BPA can disturb oxidative homeostasis through direct or indirect pathways, 
including mitochondrial function (Ooe et al., 2005), modulation of antioxidant enzymes and increase thiobarbituric acid-reactive substances in the brain of mice exposed throughout embryonic (or) fetal route (Kabuto et al., 2004). Oxidative stress has been implicated in aging and many pathological disorders, such as ischemic diseases, neurodegenerative diseases, diabetes, and cancer, although the underlying mechanisms are not always completely understood (Lenaz et al., 2012). The most meaningful studies on the effects on brain development, neuroendocrine signaling, and behavior concern the in utero and perinatal exposure to BPA. The exposure of pups or of pregnant rats or mice to BPA results in an increased expression of ERs (Ramos et al., 2003) and ER- $\alpha$ labeled neurons in diverse brain areas and in a reduced number of dopamine containing neurons (Masuo et al., 2004). CD-1 mice developmental exposure to BPA result in a significant change in the locus coeruleus (Kubo et al., 2001), while maternal exposure disrupt normal neocortical development in fetuses by accelerating neuronal differentiation (or) migration (Nakamura $e t$ al., 2006). Moreover, five studies have demonstrated low dose effects of adult BPA exposures on brain endpoints such as synapse formation and remodeling (Leranth et al., 2008). Some of these effects were observed only in response to additional hormone treatments (e.g., BPA attenuates the effects of estrogens on synapse formation (or) remodeling).

Epidemiological studies examining neurobehavioral outcomes correlates prenatal exposure to BPA exposure and neurobehavioral outcomes, such as aggressivity and hyperactivity (Braun et al., 2009) or anxious or depressed behavior with poor emotional control (Braun et al., 2011). These observations are in agreement with results of behavioral tests conducted on laboratory rodents exposed to low doses of BPA such as anxious behaviour, increase of aggressiveness, impaired learning, hyperreactivity to painful or fear-provoking stimuli, altered adult play and other sociosexual behaviors (Ryan and Vandenbergh, 2006). The intrauterine foet al life environment is critical for the normal development. The rat cerebellum during the first period after birth is considered to be equivalent to the third trimester of human pregnancy (Hamre and West, 1993; Thomas et al., 1998). Chemicals effects and changes in the levels of hormones can lead to changes in brain function and consequently in behavior (Jacobson and Jacobson, 1996; Faroon et al., 2001). Factors that affect the normal development of the cerebellum can cause pathological effects, depending on the developmental stage (Laure-Kamionowska and Maliska, 2009). To date, there have been few in vivo studies that have examined the impact of BPA exposure during gestation and lactation on brain development (Facciolo et al., 2002).

In addition, BPA $(25-50 \mathrm{mg} / \mathrm{kg} / \mathrm{day}$, i.p. injection) has been shown to enhance oxidative stress and lipid peroxidation promoting the cellular death in several organs (e.g. brain, liver, and kidney) of exposed rodents (Aydogan et al., 2008; Kabuto et al., 2003). This study was designed to assess the effect of BPA on the prenatal developmental structure of cerebellum in brain of pregnant albino rat. Therefore, the aims of this study were to illustrate the effects of BPA on the acute oxidative damage in targeted tissues and speculate the possible mechanism, by detecting the activity of the contents of MDA, and the contents of SOD, CAT, and GSH. The present study demonstrates DNA fragmentation by agarose gel electrophoresis in targeted tissues of rats exposed to low and high-doses BPA. 


\section{Materials and Methods}

Chemicals: Bisphenol-A and all chemicals were purchased from Sd Fine Chemicals, Bombay, BPA was dissolved in Sesame oil (vehicle) as stock before administration.

\section{Animals and Experimental design}

\section{Experimental animals}

Pregnant wistar albino rats weighing 250$300 \mathrm{~g}$ were used as experimental animals. The animals were obtained from the M/S Raghavendra Enterprises, Bangalore, India. They were maintained on stock diet and kept under fixed appropriate conditions of housing and handling. Different experimental groups of the animals were caged separately and an average of 6 animals per cage was maintained. The control as well as other treated groups of rats was given free access to standard chow and water ad libitum. All these animals were housed in wooden cages and provided water in glass bottles.

\section{Experimental Design}

The animals were divided into 3 groups. Animals of group (1) served as control and received a daily oral administration of sesame oil, Animals of group (2) were administered orally $50 \mathrm{mg} / \mathrm{kg}$ of BPA for 8 days and group (3) were administered orally $500 \mathrm{mg} / \mathrm{kg}$ of BPA for 8 days respectively. The doses of BPA were administered 8 days (gestational day $8^{\text {th }}$ to $15^{\text {th }}$ ). The lower dose of BPA $(50 \mathrm{mg} / \mathrm{kg})$ in this study was chosen on the base of previous studies (Bian et al., 2006; Richter et al., 2007). The weight of each animal was recorded. The rats were anesthetized with diethyl ether and sacrificing, the brain of each rat was dissected and removed and its weight was recorded. Cerebellums were dissected then fixed for histopathological examinations.

\section{Malondialdehyde Contents (MDA) Assay}

$0.5 \mathrm{~mL}$ sample dilution was removed to the test tube, and $2 \mathrm{ml}$ of $0.6 \%$ TBA solution was added. TBA solu- tion was dissolved by a small amount of $1 \mathrm{~mol} / \mathrm{L}$ sodium hyroxide, and then diluted to the concentration by $10 \%$ trichloroacetic acid. The test tube was sealed by plastic wrap with a little hole. After in the water bath boiling for 15 minutes, the test tube was cooled in the water, and then centrifuged at $10,000 \mathrm{rpm}$ for 10 minutes. The absorbance value of the supernatant was measured at $450 \mathrm{~nm}, 532 \mathrm{~nm}$ and $600 \mathrm{~nm}$ of wavelength. The formula $\mathrm{C}=6.45 \times(\mathrm{D} 532$ - D600) - $0.56 \times \mathrm{D} 450$ was used to calculated the concentration of MDA.

\section{Glutathione (GSH) Contents Assay}

Low molecular weight thiols (predominantly GSH) in cells were examined using Multiskan Spectrum, which produces a highly absorbance at $412 \mathrm{~nm}$ upon its reaction with thiols groups. $200 \mu \mathrm{L}$ of the supernatant was took and mingled with 50 $\mu \mathrm{L}$ of $10 \%$ TCA, so that the protein was precipitated, then centrifuged the liquid for the sec- ond time so as to eliminate the protein. The supernatant was diluted with PBS-EDTA, regulated $\mathrm{pH}$ with sodium hydroxide to 7.5 , and then $50 \mu \mathrm{L}$ sample solution and $150 \mu \mathrm{L}$ DTNB of $60 \mu \mathrm{g} / \mathrm{mL}$ were joined into 96 wells micro plate, kept in dark place for 5 minutes at room temperature. Finally detected the OD values at the wave- length of $412 \mathrm{~nm}$, calculate the concentration of GSH according to the standard curve.

\section{Light microscopy}

All specimens for light microscope examination were cut into small pieces and fixed in a solution of $10 \%$ formaldehyde and processed to get paraffin sections of $5 \mu \mathrm{m}$ thickness. Sections were stained with 
Haematoxylin and Eosin (H\&E) (Bancroft and Stevens, 1996). Slides were mounted using entellan and covered with cover slips prior to viewing and photography by (Nikon Eclipse E 200) light microscope.

\section{DNA fragmentation studies}

Tissue (brain) slices were placed in the digestion buffer $(10 \mathrm{mM}$ Tris- $\mathrm{HCl}$ with $\mathrm{pH}$ 8.0; 0.1 M EDTA with $\mathrm{pH} 8.0 ; 1 \%$ SDS and proteinase $\mathrm{K}$ with concentration of $1 \mathrm{mg} / 10$ $\mathrm{ml})$ and were incubated $\left(14-18 \mathrm{~h}, 55^{\circ} \mathrm{C}\right)$ in a shaking water bath. The DNA contents were extracted, precipitated, and stored as described below for the analysis by agarose gel electrophoresis. After digestion, samples were extracted three times with $10 \%$ saturated phenol/chloroform/isoamyl alcohol (24:24:1) and were precipitated using ethanol. The precipitates were rinsed two times with $70 \%$ ethanol, air dried, and resuspended in Tris EDTA buffer. DNA contents were measured using a spectrophotometer (A260/A280), and only samples with 1.8 ratios were used. Agarose gel electrophoresis was then carried out to analyze the fragmentation.

\section{Statistical Analysis}

The differences between treated samples and untreated control were evaluated by the students' T-test following a one-way analysis of variance (ANOVA) (Steel and Torrle) were performed using the Statistical Package for Social Sciences (SPSS) Package programming techniques on "Intel Core 2 Duo Processor" personnel computer. Probability values less than 0.05 were considered significant.

\section{Results and Discussion}

The results revealed more prominent signs of toxicity in rats treated with BPA, where most treated rats became less active and showed general weakness. The body and brain weights were recorded to be significantly low in the BPA treated animals as compared to their respective controls.

Effect of BPA on antioxidant enzymes (Super oxide dismutase (SOD), Catalase (CAT), Glutathione (GSH), Malondialdehyde (MDA)) in rat brain tissue

In the present study, super oxide dismutase, catalase, GSH activities in tissue of BPA treated rats (lower dose or $50 \mathrm{mg} / \mathrm{kg}$. b.w $/$ day and higher dose or $500 \mathrm{mg} / \mathrm{kg}$. b.w/day) showed significant variations $(\mathrm{P}<0.05)$, (Fig-2), when compared to control group. On treatment with BPA rats, showed significantly decreased super oxide dismutase, catalase \& glutathione activities, the gradual decreased super oxide dismutase, catalase, glutathione activities were found in the tissue of higher dose followed by lower dose BPA treated groups. Thus it is revealed that the above enzyme activities were decreased highly in higher dose of BPA treated rats when compared with control group.

The level of malondialdehyde activity in tissue of BPA treated rats (lower dose and higher dose) showed significant variations $(\mathrm{P}<0.05)$, (Fig- 2), when compared to control group. On treatment with BPA rats, showed significant elevated levels of malondialdehyde activity, the gradual increased malondialdehyde activity was found in the higher dose, lower dose BPA treated groups. Thus the above observations revealed that highly increased malondialdehyde activity in higher dose BPA treated group when compared to control group.

Histological changes in brain and cerebellum tissues

Figure-3 illustrates the histopathological assessment of brain tissue of control and experimental animals. BPA treated rats $(3.2$ 
\& 3.3) exhibited spongiform necrosis (1), marked gliosis (2), nuclear pycnosis (3), and lymphocytic inflammatory infiltrates as against normal architecture shown by the brain of control rats (Figure-3.1). Treatment with BPA toxication showed the incidence of these pathological changes in the brain tissue.

In control group cerebellum, the Purkinje cells $(\mathrm{P})$ in the third layer are arranged at the junction between the molecular layer (M) with stellate $(\mathrm{S})$ and the granular layer $(\mathrm{G})$. They are nearly mature cells. The internal granular layer is thick and contains small neurons called the granule cells, which have large, rounded nuclei and scanty cytoplasm and immature with few number of basket cells (B) (Fig-4.1). Effect of BPA low dose on the pregnant rat revealed the transverse section of cerebellum showing disruption in the Purkinjee cells layer $(\mathrm{P})$ \& cells were immature (Fig-4.3). Effect of BPA high dose on the pregnant rat revealed the transverse section of cerebellum showing highly disruption in the Purkinjee cells layer, degenerative changes with loss of their normal pyriform shaped appearance. The purkinjee cells showed a notable degree of immaturity and a much lower number of cells compared to the control (4.2) group. The cells are scattered unorganized with wide spaces and revealing signs of injury and they had lost their normal organization. Many cells were mostly pyknotic (Fig.4.4).

\section{DNA damage}

The DNA damage (brain tissue) caused in the cells as a result of BPA induction was examined by agarose gel electrophoresis is shown in Fig-5. The results indicated that DNA of BPA-induced group showed a comet tail indicating the DNA damage arising from the genotoxicity in the BPAinduced cells when compared to the DNA of control cells. Whereas intensity of DNA fragmentation is high in high dose treated sample compared to low dose treated sample. Thus DNA fragmentation gradually increased from lower (L2), higher dose (L3) and normal DNA banding pattern was observed in control (L1), (Fig-5).

Epidemiological and experimental data indicate that in utero exposure to environmental chemicals and prescribed drugs during pregnancy can mediate early embryonic losses, spontaneous abortion, fetal growth retardation and resorptions, decreased litter size, fetal malformations and low body weight and low birth weight (Khattak et al., 1999) via ROS generation which damages cellular macromolecules (Nicol et al., 2000). BPA, an environmental contaminant, is concerned as a serious threat for human health due to its wide spread in nature (Richter et al., 2007). Furthermore, exposure to BPA during neonatal and prenatal periods has been shown to led to memory impairment, sexual differentiation process and behavior in the offspring of experimental animals (Nakamura et al., 2012) and cognitive impairment in rodents (Negishi et al., 2003). It has been suggested that environmental exposure to BPA may affect the developing brain by enhancing the local biosynthesis of estrogen in the brain, inhibiting estrogen receptor beta (ERbeta) and N-methyl- D-aspartate receptor (NMDAR) expressions(Kawai et al., 2007). Furthermore, due to its estrogenic and antiandrogenic activities (Delfosse et al., 2014), BPA can interfere with the dimorphic development of the neuronal networks controlling many endocrine systems and brain functions. As a matter of fact, sex differences have been well documented not only in the mechanisms controlling reproduction, but also in non reproductive behaviors (Jazin and Cahill, 2010 and Wolstenholme et al., 2011). In this study of results revealed that, the body and brain weights were recorded to be significantly 
low in the BPA treated animals as compared to their respective controls. BPA exposure also decreases the body and brain weight, because the reason that can be attributed for the decrease in protein contents under toxic stress may be due to formation of lipoproteins, which are utilized for repair of damaged cell and cell organells. The decreased protein content may be related to impaired food intake of animals, the increased energy cost of homeostatis, detoxification mechanism, also be attributed to the destruction or necrosis of cellular function and consequent impairment in protein synthetic machinery during stress condition. Hiroi et al., (2006) reported that, BPA possesses an inhibitory effect on protein disulfide bonds formation in cells therefore it might have a potential effect in disrupting various physiological functions. The binding of BPA to human serum proteins has been reported by Csandy et al., (2002). Gopinath (Gopinath, 1984) observed that the brain weight was low in the experimental under nutrition. BPA exposure also decreases the body and brain weight dose dependant and may be due to loss of animal's appetite and activities.

The findings of the present study, also, showed that the oxidative stress rise in BPA treated rats was accompanied by concomitant decrease in the activity of some antioxidant enzymes involved in the detoxification of ROS, namely SOD, CAT as well as the level of GSH in the brain tissues comparing with the control declaring the prooxidant effect of BPA. These findings agreed with the antecedent studies of Andersen, (2004). Who showed that BPA exposure enhanced the neuronal lipid peroxidative damage with concomitant alterations in the enzymatic antioxidant defense status, thus having serious bearing on the functional and structural development of the central nervous system. Similar data recorded a decrease in the antioxidants such as GSH and SOD activity in the brain of aluminium exposed rats and human Dua $\mathrm{R}$ and Gill KD (2001). BPA could be caused by inflicting damage to membrane lipids, proteins and antioxidative enzyme defense system. However, the increased BPA concentration could deleteriously affect the neurons, leading to depletion of antioxidants and metal ions through the induction of free radicals, that exhausting SOD and CAT which function as blockers of free radical processes. These results are in accordance with Bindhmol et al., 2003 and Kabuto et al, 2004 whom recorded a significant decrease in the activities of SOD and CAT in brain of rats after BPA treatment. Alternatively, the decreased enzyme activities could be related to a reduced synthesis of the enzyme proteins as a result of higher intracellular concentrations of BPA. SOD, catalase, and Glutathione are intracellular enzymatic antioxidants, which are responsible for disposing reactive oxygen species such as hydrogen peroxide and superoxide free radicals. In this study, the basal levels of activities of these three enzymes in the BPA treated brain tissue of the rats were found to be much lower than those in the control. Typically, lipid peroxidation was the primary results of oxidative stress, and correlated effects on the levels of MDA (increased) were also observed. The toxicity of MDA is so great that it can induce the peptide chain's fracture and cross-linking of amino acids in enzyme molecule to form the polymer. As a result, the enzyme activity is renewed, lost or changed, the structure of cell membrane is being destroyed, disorderly ionic exchange is induced inside and outside of cell membrane and excessive free radicals are produced. Then cells are destroyed and the organism is also injured. The result is agreed with the ROS result. Besides, the balance of oxidants and antioxidants is very important for healthy cells. So the 
intracellular GSH level in the BPA treaded cells was monitored to further prove the formation of ROS. GSH is an important anti- oxidant that protects cells from ROS and plays a critical role in the intracellular ROS removal. Hence the depletion of intracellular GSH is usually regarded as a scientific measure of the oxidative stress. Just as illustrated in the Figure-3, the absorbance in the BPA treated cells was much weaker than the control group. In concordance with our results, $\mathrm{Wu}$ et al. showed significant decrease in the levels of GSH in BPA group; this decrease indicated tissues being damaged (Wu et al., 2011). The research before showed that ROS may be one of the major mediators in the regulation of autophagy (Wang et al., 2009), ROS is a natural by product of the normal metabolism in cells. A certain amount of ROS could function as a second message in the signal transduction of healthy cells. However, excessive ROS damages bimolecular, triggers the apoptosis pathways, and even further induces cell death; excessive ROS and oxidative stress may be the main reasons for multiple toxicities (Nel et al., 2006). Excess ROS can also damage a wide variety of cellular constituents including DNA, RNA, Proteins, sugar and lipids, thereby compromising cell viability. The result of this study is similarly with others who demonstrated that BPA can generate ROS that causes oxidative damage in the brain tissue of rats (Korkmaz et al., 2010). Treatment with BPA induced oxidative stress in various tissues of rodent (Kabuto et al., 2003) by decreasing antioxidant enzymes and increasing hydrogen peroxide and lipid peroxidation (Bindhumol et al., 2003). The presence of high level of ROS during embryonic period, the induction of oxidative stress in the target tissue has been suggested as a possible mechanism of chemical carcinogenesis (Kensler et al., 1989). Exogenous xenobiotics (BPA) and endogenous compounds can disturb this balance by enhanced generation of free radicals and decreased antioxidant capacity.

The light microscopic observations of the brain tissues BPA treated rats showed; cavitations and necrotic areas appeared in all cerebellar layers. Exposure to BPA may cause marked histophathological alterations in the brain tissue which were represented by focal as well as diffuse gliosis, spongioform necrosis and nuclear pycnosis in brain regions with neuronal degeneration. Parallel to our findings, those recorded by Mohamed Atif A. Said Ahmed et al, who found that BPA causes histopathological lesions in brain regions including neuronal degeneration as cytoplasmic vacuolization hemorrhage, ghost cell and gliosis. Our histopathological findings are correlated to those of Matyja, who noticed that exposure to chemical causes marked histopathological alteration in the cerebral cortex including neuronal degeneration, perecellular odema and gliosis. The cerebellar cortex of pregnant rat is seen to be consisted of four layers, the outermost external granular layer which covering the surface of the developing cerebellum, molecular layer, Purkinje cell layer and an inner granular formed of four to five rows of cells. The molecular layer is thin layer and immature with few number of basket cells. Tranverse section of BPA treated rat cerebellum showing disruption in the purkinjee cells layer, some Purkinje cells were small in size with abnormal shape and loss of the prominent dendrite. Also the arrangement is impaired and disorganized in the BPAtreated animals as compared to the controls (4.1).According to Brodal, the functions of certain learning and memory have been associated with different areas of the brain like the hippocampus and cerebellum. However, BPA treated group showed 
marked cell distortion groups with high level of degeneration in the cells as compared to the controls. The toxic effect of BPA during pregnancy causes different pathological changes in the cerebellum (including degenerative changes) observed in this study. Pathological changes in cerebellum (4.3 \& 4.4) of pregnant rats treated with BPA demonstrated the features of nervous tissue damage expressed by marked cellular damage. The observed changes suggested high generation of ROS in the cells. BPA can be dispersed to the organs of the body after administrated. It can also get to the brain. The brain consumes a large quantity of oxygen, making it particularly susceptible to oxidative stress Andersen (2004). The pregnant rat selected was in the time of growing development especially for the brain. Currently, we should pay attention to the application damage, as the $50 \& 500$ $\mathrm{mg} / \mathrm{kg} \cdot \mathrm{d}$ BPA administrated can induce oxidative damage especially for the brain of pregnant rat.

Table.1 Effect of BPA on average body weight \& brain weight (gm) of early gestation rat

\begin{tabular}{|c|c|c|}
\hline Group & Body weight/ 8days & Brain weight/8 days \\
\hline CONTROL- (I ) & $11.48 \pm 0.18$ & $1.08 \pm 0.05$ \\
\hline LOW DOSE(50mg/kg/d)- ( II) & $9.20 \pm 0.16$ & $0.90 \pm 0.04$ \\
\hline HIGH DOSE(500mg/kg/d)-( III) & $7.12 \pm 0.16$ & $0.60 \pm 0.04$ \\
\hline
\end{tabular}

Fig.1 Body weight and brain tissue weight of albino rats exposed to BPA.
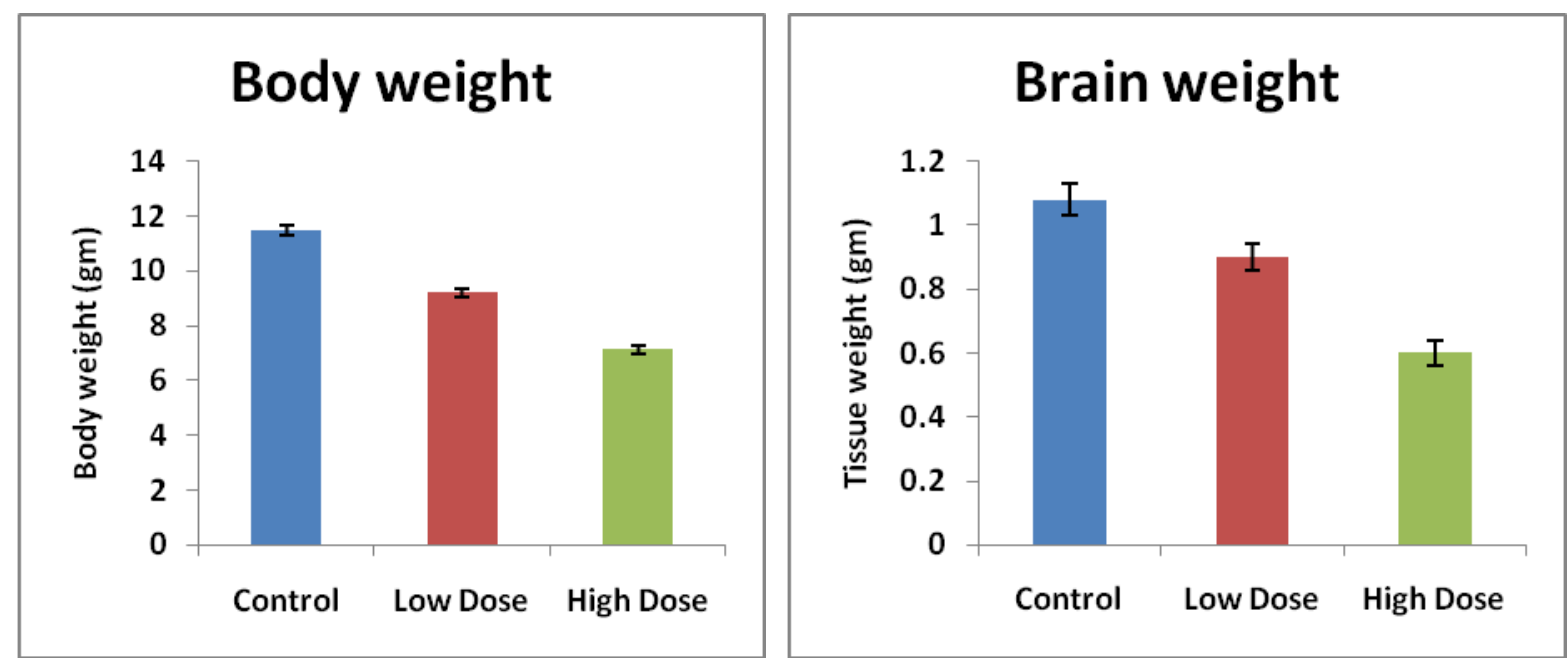
Fig.2 Superoxide dismutase, Catalase, Glutathione, Malondialdehyde activity levels in brain tissue samples of albino rats exposed to BPA

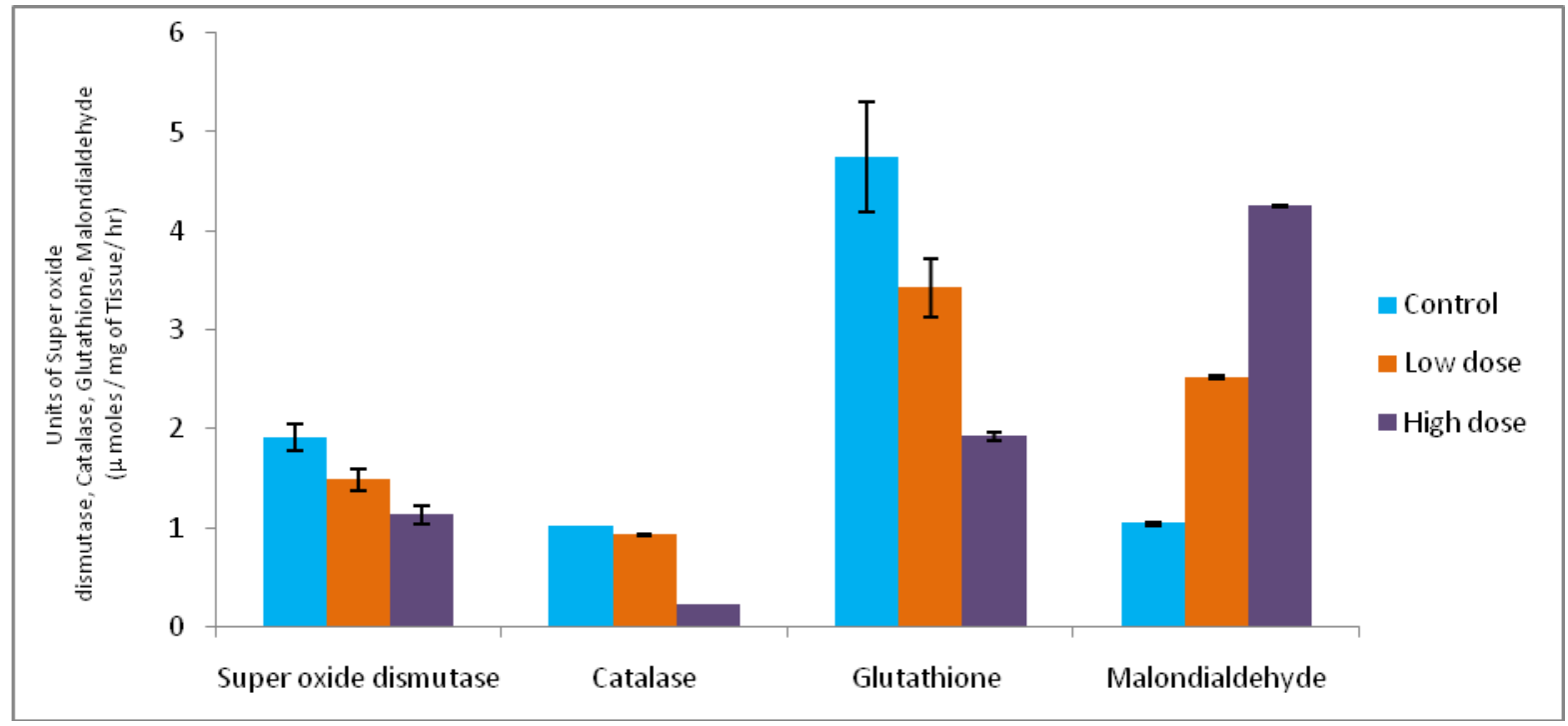

Fig.3 Brain tissue morphology (H \& E staining)

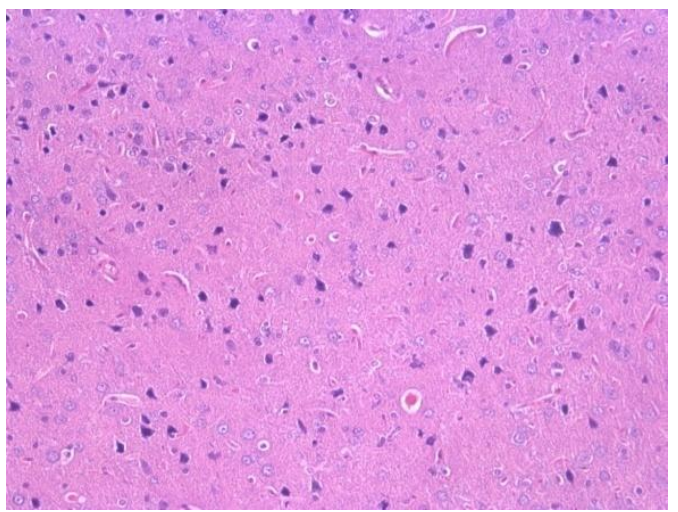

Fig-3.1: Control

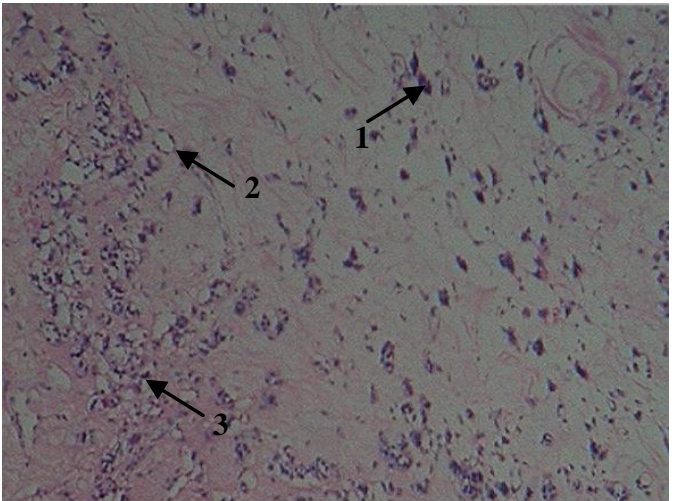

Fig-3.2: Low dose $(50 \mathrm{mg} / \mathrm{kg})$

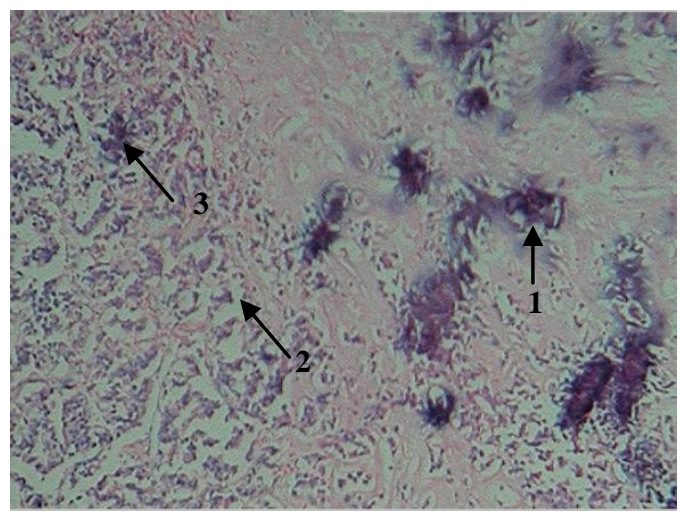

Fig-3.3: High dose $(500 \mathrm{mg} / \mathrm{kg})$ 
Fig.4 Purkinjee cell layer morphology in cerebellum tissue (H \& E staining)

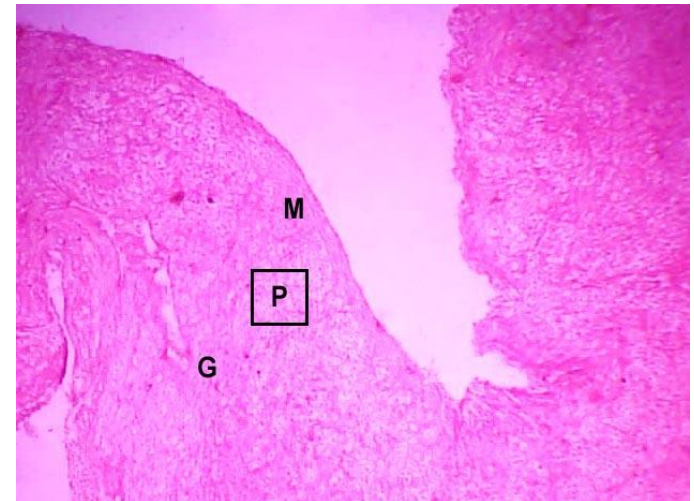

Fig-4.1: Control cerebellum

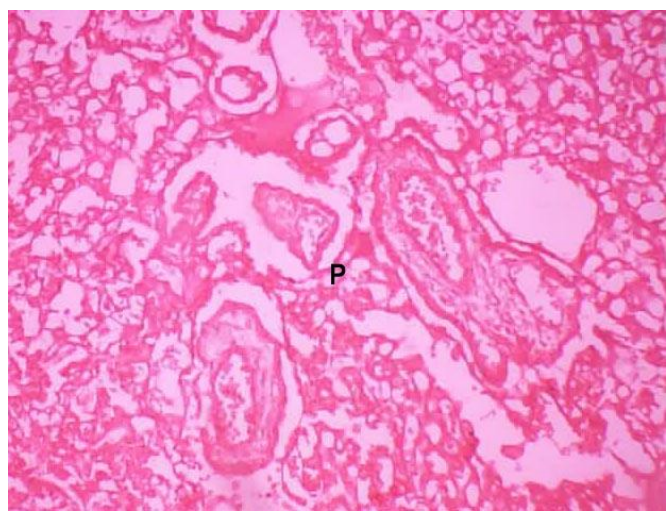

Fig-4.3: Low dose Purkinjee cell layer

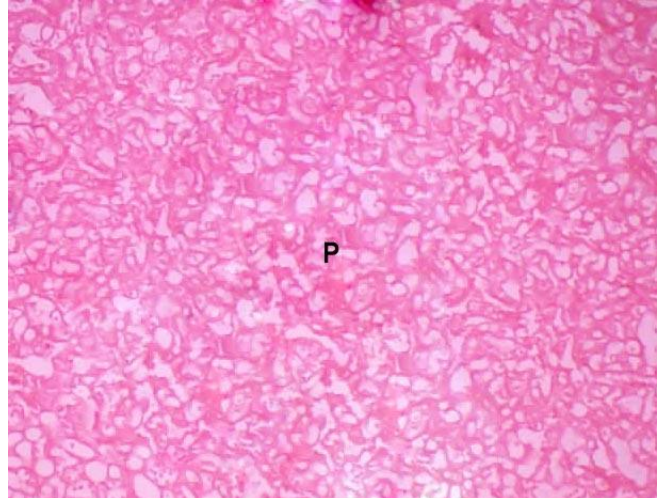

Fig-4.2: Purkinjee cell layer

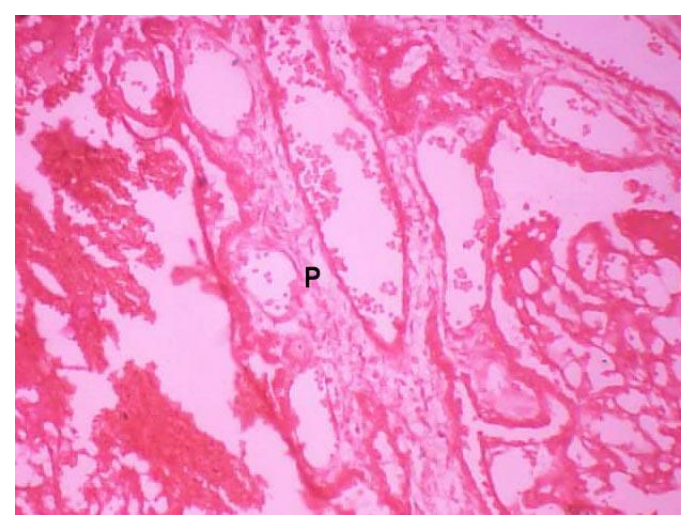

Fig-4.4: High dose Purkinjee cell layer

Fig.5 Agarose gel electrophoresis of genomic DNA extracted from rat brain from various treatment groups. Lane 1: DNA from the control group, Lane 2: DNA from the low dose treated group, Lane 3: DNA from higher dose treated group, M: marker.

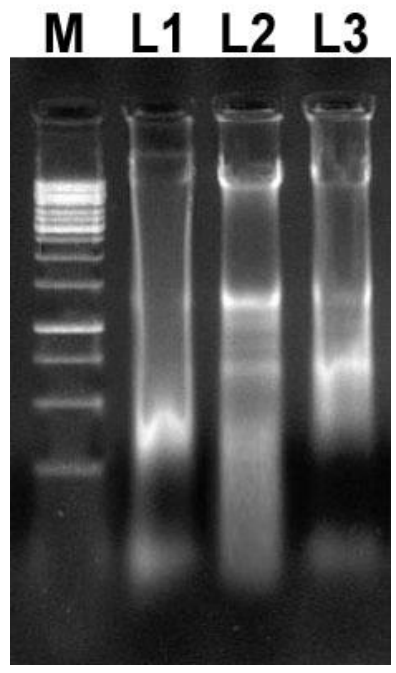


Lastly, effects of BPA toxicity on DNA were also investigated (brain tissue). BPA induction caused an increased DNA damage as indicated by the increased fragmentation of DNA were observed. DNA fragmentation in this study as a consequence of BPA exposure. BPA is known to increase the levels of reactive oxygen species (Cavalieri et al., 2010). This is known to cause damage to various macromolecules and also to DNA. Damage to DNA is one of the markers and typical characteristic of apoptosis (Wyllie, 1993) and BPA toxicity can lead to faster apoptosis as seen in the previous study which clearly revealed disruption of cells. Further, BPA has been shown to possess antioxidative properties and hence may decrease the levels of free radicals and ultimately the damage to DNA.

During critical periods of embryonic and prenatal development, the hormonal milieu is crucial for the correct organization of neuroendocrine circuits that coordinate sexspecific physiology so, the altered expression levels of hormones at the hypothalamus and pituitary (in brain) levels may be the cause and/or the consequence of the changes in gonadal steroidogenesis and sex hormone production (Fernandez et al., 2010). There are possible mechanistic effects of BPA on the local regulatory circuits of hypothalamus and pituitary (in brain) (Mahoney and Padmanabhan, 2010). Knobil et al., (1994) pointed out that the decrease serum LH level by BPA may be due to the consequence of the increase in GnRH pulse frequency, leading to desensitization of the pituitary. Additionally, decrease of serum LH might cause by estrogenic action of BPA at the level of the HPG axis, mimicking the estrogenic suppression of LH, FSH secretion, while it inhibited LH, FSH secretion, suggesting that the effect of BPA on gonadotropes is specific to the mechanism that underlies the control of LH, FSH secretion. In my previous study, decreased levels of progesterone caused by BPA could lead to low levels of the sex hormones, which will trigger impaired immune function and a host of other issues associated with hormone imbalance. BPA might disrupt the motility of muscular layers of the oviduct and the sequential phenomena of embryo implantation by binding estrogen \& progesterone receptors (Uterine proliferation and differentiation during the pre implantation period in pregnancy have been well characterized, and these processes are dependent on estrogen and progesterone), ultimately resulting in a reduction of the number of embryos and in uterine weight during pregnancy. It can leads to fetoplacental and uterine growth restriction in pregnant rats. These data indicate that BPA may be capable of altering important events during critical periods of brain development.

\section{References}

Andersen, J.K. 2004. Oxidative stress in neurodegeneration: Cause or consequence. Med, 7, 18-25.

Bancroft, J.D. and Stevens, A. 1996. Theory and practice of histological techniques. 4th ed.Churchill Livingstone: New York.

Braun, J.M., Kalkbrenner, A.E., Calafat, A.M., Yolton, K., Ye, X., Dietrich, K. N., and Lanphear, B.P. 2011. "Impact of early-life bisphenol A exposure on behavior and executive function in children," Pediatrics, vol. 128, no. 5, pp. 873-82.

Braun, J.M., Yolton, K., Dietrich, K.N., Hornung, R., Ye, X., Calafat, A.M., and Lanphear, B.P. 2009. "Prenatal bisphenol A exposure and early childhood behavior.," Environ. Health Perspect., vol. 117, no. 12, pp. 194552. 
Faroon, O., Jones, D., de Rosa, C. 2001. Effects of polychlorinated biphenyls on the nervous system. Toxicol. Ind Health; 16: 305-33.

Hamre, K.M. and West, J.R. 1993.The effects of the timing of ethanol exposure during the brain growth spurt on the number of cerebellar Purkinje and granule cell nuclear profiles. Alcohol Clin. Exp. Res., 17: 610-22.

Jacobson, J.L., Jacobson, S.W. 1996. Intellectual impairment in children exposed to polychlorinated biphenyls in utero. $N$ Engl. J. Med., 335: 7839.

Kabuto, H., Amakawa, M. and Shishibori, T. 2004. "Exposure to bisphenol A during embryonic/fetal life and infancy increases oxidative injury and causes underdevelopment of the brain and testis in mice," Life Sci., vol. 74, no. 24, pp. 2931-2940.

Kawai, K., S. Murakami, E. Senba, T. Yamanaka, Y. Fujiwara, C. Arimura, T. Nozaki, M. Takii and C. Kubo. 2007. Changes in estrogen receptors alpha and beta expression in the brain of mice exposed prenatally to bisphenol A. Regulatory Toxicol. Pharmacol., RTP, 47: 166-170.

Kensler, T.W., Egner, P.A., Taffe, B.G., and Trush, M.A. 1989. Role of free radicals in tumor promotion and progression. Prog. Clin. Biol. Res., 298, 233-248.

Khattak, S.G., Moghtader K.K., McMartin M., Barrera D., Kennedy D and Koren G. 1999. Pregnancy outcome following gestational exposure to organic solvents: a prospective controlled study. The J. American Med. Assoc., 281: 1106-1109.

Korkmaz, A., Ahbab, M.A., Kolankaya, D. and Barlas, N. 2010. Influence of Vitamin $\mathrm{C}$ on Bisphenol A, nonylphenol and octylphenol induced oxidative damages in liver of male rats. Food and Chem. Toxicol., 48: 2865-2871.

Kubo, K., Arai, O., Omura, M., Watanabe, R., Ogata, R., and Aou, S. 2003. "Low dose effects of bisphenol A on sexual differentiation of the brain and behavior in rats." Neurosci. Res., vol. 45 , no. 3, pp. 345-56.

Laure-Kamionowska, M., Mali ska, D. 2009. Calbindin positive Purkinje cells in the pathology of human cerebellum occurring at the time of its development. Folia Neuropathol., 47: 300-5.

Mohamed Atif, A., Said Ahmed and Refaat A. Eid. 2015. Effect of Bisphenol-A on the Post-Natal Development and Structure of Rat Cerebellum. Int. J. Curr. Microbiol. App. Sci., 4(6): 1435.

Nakamura, K., Itoh K., Dai H., Han L., Wang X., Kato S., Sugimoto T. and Fushiki S. 2012. Prenatal and lactational exposure to low-doses of bisphenol A alters adult mice behavior. Brain and Development, 34: 57-63.

Ramos, J., Varayoud, G.J., Kass, L., Rodríguez, H., Costabel, L., MunozDe-Toro, M. and Luque, E.H. 2003. "Bisphenol-A induces both transient and permanent histofunctional alterations of the hypothalamicpituitary-gonadal axis in prenatally exposed male rats," Endocrinol., vol. 144, no. 7, pp. 3206-15.

Ryan, B.C. and Vandenbergh, J.G. 2006. "Developmental exposure to environmental estrogens alters anxiety and spatial memory in female mice," Horm. Behav., vol. 50, no. 1, pp. 8593.

Sui, Y., Ai, N., Park, S.H., Rios-Pilier, J., Perkins, J.T., Welsh, W.J. and Zhou, C. 2012. "Bisphenol A and its analogues activate human pregnane $\mathrm{X}$ 
receptor.," Environ. Health Perspect., vol. 120, no. 3, pp. 399-405.

Thomas, J., Goodlett, Ch R., West, J. 1998. Alcoholinduced Purkinje cell loss depends on developmental timing of alcohol exposure and correlates with motor performance. Dev. Brain Res., 105: 159-66.
Wang, S.H. and Shih, Y.L. 2009. Cadmium toxicity toward autophagy through ROS-activated GSK-3beta in mesangial cells. Toxicol. Sci., 108: 124-131.

Wyllie, A.H. 1993. Apoptosis (The 1992 Frank Rose memorial lecture). $B r . J$. Cancer, 67: 205-208.

\section{How to cite this article:}

Geetharathan, T., 2016. Effect of Bisphenol- A on Brain Tissue in Pregnant Rat. Int.J.Curr.Microbiol.App.Sci. 5(8): 677-689. doi: http://dx.doi.org/10.20546/ijcmas.2016.508.077 